\title{
LA NOTA Y EL PRINCIPIO DE RACIONALIDAD DEL ORDEN MIUSICAL
}

\author{
Eva Lainsa de Tomas
}

Descartes comienza su Compendium Mrusicae refieriéndose a la música como el arte de complacer o agradar y de conmover por medio del sonido. Efectivamente, "su objeto es el sonido" y para hacer música (para alcanzar el objetivo propuesto, media ad finem) el objeto ha de delimitarse: ha de organizarse en función de determinadas características o propiedades (affeciones). ${ }^{`}$ El trabajo musical del sonido responde pues siempre a un principio de racionalidad (reflexionado y explícito o no) y por principio de racionalidad cabe entender aqui las leyes de funcionamiento interno de las obras musicales, es decir, el principio composicional que da cuenta en última instancia de las combinaciones sonoras establecidas a lo largo de la obra. 2

Es en función de tal principio de racionalidad que los sonidos se nos presentan como susceptibles de conformar una obra musical en tanto pueden identificarse, medirse y compararse entre si. En la música occidental llamada culta la posibilidad de establecer una ordenación racional del ámbito musical se ha desarrollado de manera paralela a la notación. El objeto de la música es el sonido... organizado, es decir presentado bajo forma de nota en función de una serie de características o parámetros bien definidos. Así, altura, duración, intensidad y timbre han sido las características del sonido que han permitido ordenar y clasificar el material de base con el que trabaja el compositor. La manera de ordenar los sonidos musicales así concebidos puede variar: pasar de una organización modal a una organización tonal o bien de la tonalidad al serialismo supone ciertamente un cambio en el modo de organizar el espacio sonoro ${ }^{3}$, pero lo que no parece poner en cuestión es la delimitación del material de base: los sonidos expresados bajo forma de nota.

De esta maneri, la escritura como racionalización del orden musical pasa en primer lugar por determinar qué sonidos son interesantes o significativos para el músico. De ahí la distinción que ha marcado la práctica musical occidental entre sonido y ruido y la gran polémica que se desarrolla durante el siglo $\mathrm{xx}$ en torno a la posibilidad (necesidad para algunos autores) de integrar —o más bien de no excluir a priori- el denominado sonido no organizado o "ruido". Cabría así analizar gran parte de la reflexión y de la práctica instrumental contemporáneas a la luz de esta cuestión:

Para algunos autores la posibilidad de racionalización del orden musical pasa por trabajar con un material determinado en función de parámetros o caracteristicas bien delinidas y que encuentran su correlato ${ }^{4}$ en el orden físico acústico. Para otros si de lo que se trata es de hallar

\footnotetext{
I Finis, ut delectet, variosque in nobis moreal (Iffectus.(...)

Media ad finem, rel soni affectiones duae sum praccipuae: nempe huins differentiae, in ratione dhralionis rel temporis, \& in ratione intensionis circa acumm aut grave. Compendium musicac, edición bilingle de Frédéric de Buzon, P.U.F., 1987, p.55.

2 Evidentemente, en una perspectiva mós amplia habria que preguntarse además por la legitimimación del uso de tal principio composicional que hallaria su base en la fisica o en la aritmetica, como lo hacen Zarlino, Rameau, Jetmholt2, Hindemith o Xenakis.

3 Donde por espacio sonoro nos referimos a esa espacialidad intrinseca al orden musical, un espacio fundamentalmente definido a partir de las relaciones que las alturas de los sonidos establecen entre si.

4 Su correlato para algunos autores (Boulez o Xenakis) su legitimación para otros (Rameau o, en nuestros dias, Jacques Chailley).
} 
el principio de racionalidad que da cuenta no sólo de la práctica musical occidental de los últimos ocho siglos sino del orden musical (abarcando prácticas no occidentales y toda la producción contemporánea) hay que comenzar por cuestionar nuestra concepción del material de base con el que se trabaja — con el que se puede trabajar-, de ahí importancia de las teorizaciones en torno a la música concreta y a la noción de objeto musical.

A continuación expondré dos maneras diferentes de abordar el problema de lo que he denominado la nota y el principio de racionalidad del orden musical y que en términos más técnicos se traduce en la búsqueda de técnicas de composición que permitan integrar las nuevas tecnologías a los procedimientos de escritura que constituyen el background de los compositores contemporáneos. Se trata de hacer una lectura de las propuestas lanzadas en torno a los años cincuenta-sesenta por Pierre Boulez y lannis Xenakis, tomando como hilo conductor un problema común que de alguna manera estaba ya apresente en la práctica musical anterior pero que —por razones más bien empiricas, de desarrollo tecnológico- sólo se plantea explícitamente en este momento. Estos dos compositores tienen, por otra parte, en común su reivindicación de lo que podriamos denominar una actitud cartesiana o racionalista ante la música.

Efectivamente, la reflexión en torno a la nota como principio organizativo ya se trata de modo implicito en la obra de los vanguardistas o "bruitistas" a comienzos de siglo aunque la reflexión propiamente dicha tenga lugar en torno a los anos cincuenta en Francia y Aleminia. Es la época en que se publica el Tratado de los objetos musicales de Pierre Schaefier y Pensar la misica hoy de Pierre Boulez ${ }^{5}$ En una reciente entrevista, a propósito de la redición de este escrito, Pierre Boulez recuerda el contexto en que pareció necesario publicar esta serie de conferencias impartidas en los célebres cursos de verano de Darmstadt. Comenzaba la década de los sesenta y el compositor alude a la práctica composicional occidental como bloqueada en un trabajo de investigación sin fin. Pesaba como una losa la sombra de la Escuela de Viena, Schönberg y Webern, fundamentalmente. El nuevo orden serial habia sido sistematizado por René Leibowitz dando lugar a una estética demasiado uniforme que la generaciön de Boulez intentaba sobrepasar. Las nuevas tecnologias representadas en principio por la electroacústica parecian heredar el trabajo de los vanguardistas de principio de siglo y se presentaban como un campo abierto a nuevas posibilidades siempre con la perspectiva de alcanzar por fin el anlelado continum sonoro. Se notaba igualmente la influencia (ya adivinada en Debussy) de concepciones y prácticas musicales orientales. Pero lo que treinta años más tarde Boulez recuerda como la nota dominante del momento es un estado de dispersión, en el que sólo se busciban soluciones a corto plazo. De ahi la necesidad de recopilar una serie de trabajos en los que se trató de buscar una racionalidad global que no obstante comportara un sistema lo suficientemente ceñido como para evitar la desintegración inevitable del material.

Así, el capitulo dedicado a la técnica musical de Pensar la misica hoy obedece a la búsqueda de un sistema que permitiera formas de escritura menos estrictas que las directamente heredadas de la Escuela de Viena, es decir, que no dependieran directamente del sistema, lo que equivale a admitir la posiblidad de ampliaciones o resctricciones muy personalizadas del mismo. Un primer punto en común con Pierre Schaefter o con Xenakis: "comenzar por establecer nociones primitivas...". Esta manera de retomar el vocabulario desde el principio, desde sus fundamentos responde a una preocupación común: no reflexionar únicamente sobre la

5 El Traité des objets musicaur (Éditions du seuil, Paris) se publica en 1966. Penser la musique aujourd'hui (Gallimard, Paris) en 1963, La crise de la musique sérielle (Granesaner Blaner $n^{\circ} 1$ ) en 1956, Mhusiques formelles (La Revue Afusicale n²53-254, Paris, Richard Masse) en 1963. 
manera de servirse de las lerramientas, sino sobre las herramientas mismas con las que se cuenta (lo que en el Tratado de los objetos musicales se llevará hasta las últimas consecuencias: reflexionar musicalmente sobre las herramientas mismas y sobre el material mismo). De ahi la importancia de re-definir como nociones primitivas la de serie por una parte y la de fenómeno sonoro por otra. La posibilidad de ampliar la noción de serie a todos los parámetros parte para Boulez de la definición de serie como germen de determinada jerarquización del material. Alrora bien, esta jerarquización vendrá determinada por "ciertas propiedades psico-físiológicas acústicas", es decir, dependerá del fenómeno sonoro mismo y sirve para organizar - deducirun conjunto finito de posibilidades - de combinaciones- sonoras. ${ }^{6}$ Esta idea de que es posible ampliar la noción de serie y aplicarla a todos los parámetros sonoros se plantea paralelamente a una redefinición del fenómeno sonoro que Boulez concibe en términos espaciales: el espacio sonoro musical se piensa en cuatro dimensiones (altura, duración, intensidad y timbre) más la categoria espacial evidente que denominamos el espacio en general. Cuando habla de la ampliación de la noción de serie a todos los parámetros Boulez no sólo admite la posibilidad de trabajar con diferentes categorias de espacio (espacio de las alturas -el más evidente-, el de los tiempos, el de los timbres o el dinámico) sino que está exigiendo un tratamiento diferente para cada espacio y sobre todo un respeto hacia la especificidad de cada uno de estos espacios cuando se trata de la configuración global de la estructura musical. Y aqui el problema que se plantea es evidentemente, cómo integrar en la composición aquellos parámetros que se presentan como menos evidentes o menos "manipulables" que, por ejemplo, la altura. O esta misma noción cuando el objeto que se está manipulando no es susceptible de ordenación en función de las caracteristicas de la nota instrumental (que entre otras propiedades posee la de tener una altura determinada en el marco de una escala temperada). De alí la clasificación general de los espacios como lisos y estriados, aplicable tanto al ambito de las alturas como al del tiempo al lacer intervenir al tempo (tiempo pulsado - estriado-y tiempo amorfo - liso-). Curiosamente en la época en que se escribe Pensar la misica hoy la solución que busca Boulez al problema de la integración de las posibilidades técnicas entonces incipientes a la escritura parece $-y$ asi se ha interpretado tópicamente- llevarle a apostar por la escritura al constatar la imposibilidad de realizar en la práctica los espacios lisos y estriados. Como reconocerá treinta años más tarde, las posibilidades abiertas por la informática han ilustrado en el terreno de la composición la teoría del espacio sonoro establecida en 1963. Baste con citar Réponse como ejemplo en este sentido, una obra en la que el espacio fisico o empirico juega un papel determinanate en la configuración misma del espacio intrinseco de la obra.

Otra manera de "racionalisno musical" o búsqueda de un sistema desde el cual integrar tecnología y escritura es la concepción estoccistica que propone Iannis Xenakis en torno a la misma época en que Boulez recopila Pensar la misica hoy.

Frente a la herencia omnipresente de la Escuela de Viena, Xenakis reacciona de manera drástica con la publicación en 1955 de La crisis de la música serial ${ }^{7}$ donde denuncia la ininteligibilidad de composiciones basadas en sutiles organizaciones seriales imperceptibles para el oido, afirmando que la polifonia lineal terminaria por destruirse a si misma precisamente a causa del grado de complejidad alcanzado: "linalmente uno no oye nada más que un amasijo de notas de diferentes registros". El compositor recuerda quince años más tarde que liente a las contradicciones del pensamiento lineal propuso entonces un universo de masas sonoras, de vas-

6 Penser la musique... pp. 35 ss.

7 Gravesaner Blatter, op. cit. 
tos conjuntos de acontecimientos sonoros, de nubes, de galaxias regidas por características nuevas, tales como la densidad, el grado de orden, la velocidad de cambio, etc. que necesitaban definiciones y puestas en práctica que recurrieran al cálculo de probabilidades. Así nació la música estocástica, como una propuesta, en definitiva de trabajar no ya con notas aisladas sino con masas sonoras. Una concepción que Xenakis considera más general que la lineal polifónica, puesto que la englobaba como un caso particular (reduciendo las condensaciones de mubes), por más que no se tratara - todavia- de una armonia general. ${ }^{8}$ Se trata en definitiva de adaptar diferentes trabajos cientificos sobre el cálculo de probabilidades, la estadisitica y el azar a la composición musical. Con esta propuesta la composición musical se entiende como un proceso de transformaciones graduales, como la posibilidad de hacer pasar el material de manern global de un estado sonoro a otro (pudiendo pasar, por ejemplo, de la continuidad - glissando- a la discontinuidad - pizzicato; de la inmobilidad al movimiento o de un ritmo regular a un ritmo aleatorio - del orden al desorden).

Al igual que Boulez Xenakis parece experimentar la necesidad de una teorización global que de cuenta de las investigaciones y las soluciones halladas "a corto plazo", papel que parece jugar la teoria de las cribas tal como es presentada en Musiques formelles (1963) y al igual que Boulez experimenta la necesidad de comenzar por una cierta tabla rasa, por una redefinción del fenómeno sonoro. Asi en Théorie des probabilités el composition mulsicale ${ }^{9}$ Xenakis distingue entre sonidos puntuales, "granulares" (aquellos susceptibles de ser descompuestos en parámetros o variables independientes) y los sonidos de variación continua o "deslizados" (glissandi). Evidentemente, si se considera la posibilidad de trabajar de manera rigurosa y sistemática con este segundo tipo de sonidos resulta dificil la caracterización bajo forma de nota debido al aspecto discreto que ésta conlleva (uno la representaría efectivamente, como un punto en el espacio sonoro). Dado que los sonidos de tipo "deslizados" pueden ser asimilados sensorialmente a la noción fisica de velocidad, Xenakis propone una representación vectorial de los mismos. ${ }^{10} \mathrm{El}$ tamaño del vector vendrá dado por la hipotenusa del triángulo rectángulo cuyos dos otros lados serán la duración y el intervalo de altura recorrido. La velocidad dependerá asi de dos parámetros: intervalo de las alturas recorridas y duración: $\quad v=\frac{h}{f}$

Ello permitirá operar matemáticamente con los sonidos deslizados. Por otra parte, los sonidos tradicionales (cxpresados hasta entonces bajo forma de nota) no serán sino casos particulares de velocidad cero. Importa hacer lincapié en el hecho de que no tratamos con notas sino con vectores y que es preciso re-definir los principios que rijen la composición, puesto que ya no se trata de agrupar notas sino de organizar velocidades sin renunciar por supuesto a un principio de racionalidad. En este contexto Xenakis aplica la teoria cinética de los gases (ley de Maxwell) a la composición en Pithoprakta. "l El principio es el siguiente: Las investigaciones de Boltzmann y Marwell (fin del s. XIX) cuanto más caliente está un gas mayor es la agitación de las moléculas que lo componen. Esta agitación proviene de las velocidades de las moléculas que se desplazan en todas direcciones. Cada molécula se mueve a una velocidad particular que no es posible determinar individualmente lo que sí se sabe es que cuando se calienta un volúmen deternina-

8 Jers une metanusique, Missique, Architecture, Paris, Casterman, 1976, pp. 38-70.

9 Musigue, Archirecture, pp. 9-15.

10 "Theorie des probabilités es composition musicale" Musique. Architecture, p. 12.

11 Otros principios empleados en la musica estocástica son la fórmula de Poisson y las cadenas de Markov a fin de introducir en la dialśctica composicional una noción de azar controlado. 
do de gas aumenta el conjunto de las velocidades de las moléculas de manera proporcional a la temperatura. La fómula de Boltzmann y Maxwell nos da la probabiliad de existencia de una cierta velocidad v para una temperatura dada, $t$. Es decir, que permite estimar a qué temperatura esta velocidad v será la de un determinado procentaje de las moléculas presentes:

$$
f(v)=\frac{0.2}{1 \sqrt{\pi}} \cdot e^{-\frac{t^{2}}{r^{2}}} \text {, presentes: donde } e \text { es el logaritmo neperiano (base } 2,71828 \ldots \text { ) y obte- }
$$
niendo la probabilidad con un número entre 0 y 1 se obtiene el porcentaje multiplicando $f(v)$ por 100.

Esta fórmula proporciona asi pues la probabilidad de una velocidad a partir de la elección de esta velocidad y de una cierta temperatura.

En M/usiques formelles Xenakis ejemplifica la aplicación de esta fórmula en relación a los glissandi de Métastasis, donde cada instrumento de cuerda efectua glissandi según una velocidad dada. Se trata entonces de determinar una ley que rija las variaciones experimentadas por las 61 partículas sonoras (los glissandi de los 61 instrumentos de cuerda) durante un tiempo dado, eligiendo una temperatura sonora determinada.

Xenakis evoca como ejemplo los compases 309 a 313 de Métastasis, donde el $6^{\circ}$ violín desciende 18 semitonos en I compás (c 311 ). Como cada compás dura I,2 segundos la velocidad del glissando será de $18 / 1,2=15$ semitonos por segundo.

Las ley de Maxwell permite igualmente obtener las probabilidades de una velocidad para una temperatura determinada. Con ello se sabe qué proporciones de sonidos deberán tener tal o tal velocidad para que el conjunto de los sonidos afectados por esas velocidades alcance la "temperatura" (grado de agitación de la masa sonora) deseada.*

\footnotetext{
- (trabajo realizado en el marco de la beca de investigación BFI 92-147 del Gobierno Vasco (UPV. San Sebastian/RCAM, Paris) y del Proyecto de lnvestigación M1EC 009.230.-0455/92).
} 